\title{
Effect of Intermediate Toxic Product on the Survival of a Resource Dependent Species: A Modeling Study
}

\author{
Ram Naresh ${ }^{1, *}$, Shyam Sundar ${ }^{2}$ \\ ${ }^{1}$ Department of Mathematics, H. B. Technological Institute, Kanpur, 208002, India \\ ${ }^{2}$ Department of Mathematics, P.S. Institute of Technology, Bhauti, Kanpur, 208020, India
}

\begin{abstract}
A nonlinear model is proposed and analysed to study the effect of intermediate to xic product on the survival of a resource dependent species in a polluted environment. It is assumed that when resource biomass uptakes pollutants/toxicants, a liquid (sap) present in the body of biomass reacts with such toxicants and as such intermediate toxic product is formed. This to xic substance then affects the biomass and the species dependent on it. The analysis of the model shows that with increase in the cumulative emission rate of toxicants in the atmosphere, the densities of resource biomass and the species dependent on it decrease and attain their lowest equilibrium. If the rate of emission of toxicants is large enough, the resource biomass may become extinct under certain conditions and the species dependent on it may not survive. The model analysis also suggests that if the formation of intermediate toxic product is restricted by way of controlling the emission of toxicants in the environment, the resulting growth of resource biomass would lead to survival of species dependent on it.
\end{abstract}

Keywords Resource Biomass, Resource Dependent Species, Intermediate Toxic Product, Stability, Numerical Simulation

\section{Introduction}

Various kinds of pollutants/toxicants discharged into the environment very often affect the resources and the species dependent on them[1-14]. In particular, Freedman and Shukla[1] studied the effect of a toxicant on single species and predator-prey systems. They have assumed that the intrinsic growth rate of species decreases with increase in uptaken concentration of toxicant whereas its carrying capacity decreases with the environmental concentration of toxicant. The effect of to xicants on a two species competitive system was studied by Chattopadhyaya[2]. Samanta and Matti[3] studied the effect of to xicant on a single species by considering the three cases of toxicant emission i.e. instantaneous spill, constant emission and rapidly fluctuating random emission of toxicant into the environment. It was shown that the toxicant concentration emitted instantaneous ly would not be sufficient to kill the population whereas the constant emission makes the population to settle down to steady state. In the third case (rapidly fluctuating random emission), the stability (local) of the system would depend on the washout rate of toxicant from the environment.

In recent years, some mathematical models have also been proposed to study the existence and survival of resource

* Corresponding author:

ramntripathi@yahoo.com (Ram Naresh)

Published online at http://journal.sapub.org/ajcam

Copyright (C) 2012 Scientific \& Academic Publishing. All Rights Reserved dependent species living in a polluted environment [15-21]. Dubey and Hussain[15] proposed models for the survival of two competing species dependent on a resource in an industrial environment. They assumed competing species to be partially dependent, wholly dependent or predating on the resource biomass. They concluded that an appropriate level of resource biomass can be maintained to ensure the survival of species if suitable efforts to conserve the resource biomass and to control the undesired label of industrialization pressure are made. Shukla et al.[16] studied a model for survival of resource dependent population to see the effect of toxicant emitted from external sources as well as formed by its precursors. They have shown that the densities of resource and the population decrease as the cumulative emission rate of environ mental to xicant increases.

It is pointed out here that in the above studies, the effect of toxicant on resource biomass and species dependent on it is considered but without incorporating the process of formation of intermediate toxic product. However, in real situations the intrinsic growth of resource biomass is, very often affected by intermediate to xic product which is formed inside the resource biomass due to some metabolic reactions. This resource biomass affected by the intermediate toxic product then affects the species dependent on it. In this direction, Naresh et al.[22] presented a mathematical model to study the effect of intermediate toxic product formed by uptake of a toxicant on plant biomass. They have shown that, as the rate of emission of to xicant increases, the equilibrium label of plant bio mass decreases. Since the toxicants emitted 
into the atmosphere are uptaken by the resource biomass, an intermediate toxic product is formed which then affects the growth of resource biomass and the species dependent on it. Hence, in the present investigation, our ma in purpose is to study the effect of intermediate to xic product on the survival of resource dependent species. Thus, we propose a nonlinear mathematical model to see the effect of intermediate toxic product on the resource biomass and on the survival of species dependent on it[22].

The paper is organized as follows, in Section 2 we present the mathematical model. The equilibrium analysis is carried out in Section 3 and in Section 4, the stability analys is of the model is presented. In Section 5, we present the numerical simulations of the model and conclusions are provided in Section 6 .

\section{Mathematical Model}

The following assumptions have been made in the modeling process,

1. The densities of resource and species dependent on it are governed by logistic models.

2. The growth rate of resource biomass decreases with increase in the concentration of intermediate to xic product.

3. The gro wth rate of resource dependent species increases with increase in the density of resource biomass.

4. The carrying capacities of resource biomass as well as that of species dependent on it decrease with environmental concentration of toxicants.

Let $B(t)$ be the resource biomass density, affected by toxicants emitted at a constant rate $Q$ in the environment. It is assumed that, when the amount of toxicants uptaken by resource biomass interacts with the bio fluid (sap) present inside the biomass, an intermediate toxic product is formed which affects the growth of resource biomass. Let $N(t)$ be the density of resource dependent species, its growth rate $r_{2}(B)$ is enhanced by the resource biomass density. Let $T(t)$ be the cumulative concentration of toxicants in the environment with natural depletion rate $\delta$ and $\alpha$ is the depletion rate coefficient of toxicants due to uptake by resource biomass. The environmental concentration of toxicants affect the carrying capacities $K_{1}(T)$ and $K_{2}(T)$ of the resource biomass and the resource dependent species respectively. It is assumed that the uptake of the toxicants by the resource biomass is directly proportional to the density of resource biomass and the concentration of toxicants. Let $U(t)$ be the concentration of toxicants uptaken by the resource biomass with $\delta_{1}$ as natural depletion rate coefficient and $U_{1}(t)$ be the concentration of intermediate toxic product formed with a rate $\alpha_{1}$ and $\alpha_{0}$ as its natural depletion rate coefficient.

Keeping in view of the above assumptions and considerations, the system dynamics is assumed to be governed by the following nonlinear ordinary differential equations,

$$
\begin{gathered}
\frac{d B}{d t}=r_{1}\left(U_{1}\right) B-\frac{r_{10} B^{2}}{K_{1}(T)} \\
\frac{d N}{d t}=r_{2}(B) N-\frac{r_{20} N^{2}}{K_{2}(T)} \\
\frac{d T}{d t}=Q-\delta T-\alpha B T+\pi \nu U B+\theta_{1} \delta_{1} U \\
\frac{d U}{d t}=\alpha B T-\delta_{1} U-\nu U B \\
\frac{d U_{1}}{d t}=\alpha_{1} U-\alpha_{0} U_{1}-v_{1} U_{1} B \\
N(0)=N_{0}>0, B(0)=B_{0}>0, T(0) \geq 0, \\
U(0) \geq 0, U_{1}(0)>0
\end{gathered}
$$

The uptaken concentration of toxicants and the concentration of intermediate to xic product are also assumed to be depleted by an amount $v U B$ and $v_{1} U_{1} B$ respectively due to falling of biomass on the ground. A fraction of the depleted amount $v U B$ (i.e. $\pi v U B$ ) may also re-enter the environment, thus increasing the growth of toxicants. The constants $0 \leq \pi \leq 1$ and $0 \leq \theta_{1} \leq 1$ are reversible rate coefficients. All the constants are assumed to be non- negative.

In the model, the function $r_{1}\left(U_{1}\right)$ denotes the intrinsic growth rate of resource biomass which decreases as the concentration of intermediate toxic product $U_{1}$ increases and hence, we assume that,

$$
r_{1}(0)=r_{10}>0, r_{1}^{\prime}\left(U_{1}\right)<0 \text { for } U_{1}>0
$$

The function $K_{1}(T)$ denotes the carrying capacity of resource biomass which decreases as the concentration of toxicant $T$ increases and hence,

$$
K_{1}(0)=K_{10}>0, K_{1}^{\prime}(T)<0 \text { for } T>0
$$

The function $r_{2}(B)$ denotes the intrinsic growth rate of resource dependent species which increases as the resource biomass density $B$ increases and hence,

$$
r_{2}(0)=r_{20}, r_{2}^{\prime}(B)>0 \text { for } B \geq 0
$$

The function $K_{2}(T)$ denotes the carrying capacity of resource biomass which decreases as the concentration of toxicant $T$ increases and hence,

$$
K_{2}(0)=K_{20}>0, K_{2}^{\prime}(T)<0 \text { for } T>0
$$

\section{Equilibrium Analysis}

The model (1) - (5) has the following four equilibria namely,

1. $E_{0}\left(0,0, \frac{Q}{\delta}, 0,0\right)$

2. $E_{1}(0, \bar{N}, \bar{T}, 0,0)$, where $\bar{T}=\frac{Q}{\delta}$ and $\bar{N}=K(\bar{T})$

3. $E_{2}\left(\tilde{B}, 0, \tilde{T}, \tilde{U}, \tilde{U}_{1}\right)$

4. $E^{*}\left(B^{*}, N^{*}, T^{*}, U^{*}, U_{1}^{*}\right)$

The existence of $E_{0}$ and $E_{1}$ is obvious. 


\subsection{Existence and Uniqueness of $\mathrm{E}_{2}$}

The positive solution of variables in equilibrium $E_{2}$ is given by the following equations which are obtained by putting the right hand sides of model equations (1) - (5) to zero

$$
\begin{gathered}
B=\frac{r_{1}\left(U_{1}\right) K_{1}(T)}{r_{10}} \\
Q-\delta T-\alpha B T+\pi \nu U B+\theta_{1} \delta_{1} U=0 \\
\alpha B T-\delta_{1} U-v U B=0 \\
\alpha_{1} U-\alpha_{0} U_{1}-v_{1} U_{1} B=0
\end{gathered}
$$

From eqs. (7) and (8) we have,

$$
\begin{aligned}
T & =\frac{Q\left(\delta_{1}+v B\right)}{\delta\left(\delta_{1}+v B\right)+\left(1-\theta_{1}\right) \alpha \delta_{1} B+(1-\pi) \alpha v B^{2}} \\
& =f(B)(\text { let })
\end{aligned}
$$

From eq. (8) we get,

$$
U=\frac{\alpha B f(B)}{\delta_{1}+v B}=g(B)(l e t)
$$

From eq. (9) we get,

$$
U_{1}=\frac{\alpha_{1} U}{\alpha_{0}+v_{1} B}=\frac{\alpha_{1} g(B)}{\alpha_{0}+v_{1} B}=h(B)(\text { let })
$$

Using eq. (6) we as sume that,

$$
F(B)=r_{10} B-r_{1}\left(U_{1}\right) K_{1}(T)
$$

which gives

$$
F(B)=r_{10} B-r_{1}\{h(B)\} K_{1}\{f(B)\}
$$

From eq.(13), it can be seen that

$$
F(0)=-r_{1}(0) K_{1}(f(0))<0
$$

and

$$
\begin{aligned}
F\left(K_{10}\right)= & r_{10} K_{10}-\left[r_{1}\{h(B)\}\right]_{B=K_{10}} \\
& x\left[K_{1}\{f(B)\}\right]_{B=K_{10}}>0
\end{aligned}
$$

also

$$
\left.F^{\prime}(B)=r_{10}-\left[r_{1} K_{1}^{\prime}(T) f^{\prime}(B)+K_{1} r_{1}^{\prime}\left(U_{1}\right) h^{\prime}(B)\right\}\right]
$$

From eqs. (14) and (15) it is clear that there exist a root $\tilde{\mathrm{B}}$ of $F(B)=0$ in $0 \leq B \leq K_{10}$. The root will be unique, provided,

$$
\left.r_{10}-\left[r_{1} K_{1}^{\prime}(T) f^{\prime}(B)+K_{1} r_{1}^{\prime}\left(U_{1}\right) h^{\prime}(B)\right\}\right]>0
$$

where,

$$
\begin{gathered}
h^{\prime}(B)=\frac{\alpha_{1}}{\left(\alpha_{0}+v_{1} B\right)^{2}}\left[\left(\alpha_{0}+v_{1} B\right) g^{\prime}(B)-v_{1} g(B)\right] \\
g^{\prime}(B)=\frac{\alpha_{1}}{\left(\delta_{1}+v B\right)^{2}}\left[\left(\delta_{1}+v B\right) B f^{\prime}(B)+\delta_{1} f(B)\right] \\
f^{\prime}(B)=-\frac{Q\left[\delta_{1}^{2}\left(1-\theta_{1}\right) \alpha+(1-\pi) \alpha v^{2} B^{2}+2(1-\pi) \delta_{1} \alpha v B\right]}{\left[\delta\left(\delta_{1}+v B\right)+\left(1-\theta_{1}\right) \delta_{1} \alpha B+(1-\pi) \alpha v B^{2}\right]} \\
<0
\end{gathered}
$$

Knowing the value of $\tilde{B}$, the values of $\tilde{T}, \tilde{U}$ and $\tilde{U}_{1}$ can be found from eqs. (10), (11) and (12) respectively.

\subsection{Existence and Uniqueness of $E^{*}$}

The positive solution of variable in $E^{*}$ is given by the following algebraic equations,

$$
\begin{gathered}
B=\frac{r_{1}\left(U_{1}\right) K_{1}(T)}{r_{10}} \\
N=\frac{r_{2}(B) K_{2}(T)}{r_{20}} \\
T=\frac{Q\left(\delta_{1}+v B\right)}{\delta\left(\delta_{1}+v B\right)+\left(1-\theta_{1}\right) \alpha \delta_{1} B+(1-\pi) \alpha v B^{2}}=f(B) \\
U=\frac{\alpha B f(B)}{\delta_{1}+v B}=g(B) \\
U_{1}=\frac{\alpha_{1} U}{\alpha_{0}+v_{1} B}=\frac{\alpha_{1} g(B)}{\alpha_{0}+v_{1} B}=h(B)
\end{gathered}
$$

As before, let $F(B)=r_{10} B-r_{1}\{h(B)\} K_{1}\{f(B)\}$

From this, it can be seen that $F(0)<0$ and $F\left(K_{10}\right)>0$ showing the existence of a root $B^{*}$ of $F(B)=0$ in $0 \leq B \leq K_{10}$ and the root will be unique, provided,

$$
\left.r_{10}-\left[r_{1} K_{1}^{\prime}(T) f^{\prime}(B)+K_{1} r_{1}^{\prime}\left(U_{1}\right) h^{\prime}(B)\right\}\right]>0
$$

Knowing the value of $B^{*}$, we can find the values of $N^{*}, T^{*}, U^{*}$ and $U_{1}^{*}$ from eqs. (18), (19), (20) and (21) respectively.

In the following we analyse the stability behavior of above equilibria.

\section{Stability Analysis}

In this section, we describe stability analysis of different equilibria.

Theorem 1

(i) Equilibria $E_{0}\left(0,0, \frac{Q}{\delta}, 0,0\right), \quad E_{1}(0, \bar{N}, \bar{T}, 0,0)$ and $E_{2}\left(\tilde{B}, 0, \tilde{T}, \tilde{U}, \tilde{U}_{1}\right)$ are unstable.

(ii) If the following inequalities hold,

$$
\begin{gathered}
{\left[\left(\alpha T^{*}+\pi v U^{*}\right)+\frac{r_{10} B^{* 2}}{\left[K_{1}(T)\right]^{2}}\left\{-K_{1}^{\prime}\left(T^{*}\right)\right\}\right]^{2}<\frac{1}{3} \frac{r_{10} B^{*}}{K_{1}\left(T^{*}\right)}\left(\delta+\alpha B^{*}\right)} \\
{\left[\left\{-r_{1}^{\prime}\left(U_{1}^{*}\right)\right\} B^{*}+v_{1} U_{1}^{*}\right]^{2}<\frac{1}{2} \frac{r_{10} B^{*}}{K_{1}\left(T^{*}\right)}\left(\alpha_{0}+v_{1} B^{*}\right)} \\
{\left[\left(\pi v B^{*}+\theta_{1} \delta_{1}\right)+k_{3} \alpha B^{*}\right]^{2}<\frac{4}{9} k_{3}\left(\delta+\alpha B^{*}\right)\left(\delta_{1}+v B^{*}\right)} \\
\alpha_{1}^{2}<\frac{2}{9} \frac{r_{10} B^{*}\left(\delta_{1}+v B^{*}\right)^{2}\left(\alpha_{0}+v_{1} B^{*}\right)}{K_{1}\left(T^{*}\right)\left(\alpha T^{*}+v U^{*}\right)^{2}}
\end{gathered}
$$

where $k_{3}<\frac{1}{3} \frac{r_{10} B^{*}\left(\delta_{1}+v B^{*}\right)}{K_{1}\left(T^{*}\right)\left(\alpha T^{*}+v U^{*}\right)^{2}}$

then $E^{*}$ is locally asymptotically stable (See Appendix-A for proof).

To establish the nonlinear asymptotic stability of $E^{*}$, we need the bounds of different variables. For this we propose 
the following region of attraction, stated without proof Freed man and So[23].

Lemma 1 The set

$$
\Omega=\left\{\begin{aligned}
\left(B, N, T, U, U_{1}\right): 0 & \leq B \leq K_{10}, 0 \leq N \leq N_{c}, \\
0 & \leq T+U \leq \frac{Q}{\delta_{m}}, 0 \leq U_{1} \leq \frac{\alpha_{1} Q}{\alpha_{0} \delta_{m}}
\end{aligned}\right\}
$$

attracts all solutions initiating in the interior of non-negative octant, where $N_{c}=\frac{r_{2}\left(K_{10}\right) K_{2}\left(f\left(K_{10}\right)\right)}{r_{20}} \quad$ and $\delta_{m}=\min \left(\delta, \delta_{1}\left(1-\theta_{1}\right)\right)$.

\section{Theorem 2 .}

Let $K_{1 m} \leq K_{1}(T) \leq K_{10}, K_{2 m} \leq K_{2}(T) \leq K_{20}, 0 \leq-r_{1}^{\prime}\left(U_{1}\right) \leq p$, $0 \leq r_{2}^{\prime}(B) \leq r, \quad 0 \leq-K_{1}^{\prime}(T) \leq q, 0 \leq K_{2}^{\prime}(T) \leq s, \quad\left|\xi_{1}\left(U_{1}\right)\right| \leq p$, $\left|\xi_{2}(B)\right| \leq r,\left|\eta_{1}(T)\right| \leq \frac{q}{K_{1 m}{ }^{2}},\left|\eta_{1}(T)\right| \leq \frac{s}{K_{2 m}{ }^{2}}$

satisfying in $\Omega$ for some constants $K_{1 m}, K_{2 m}, K_{10}, K_{20}, p, q, r, s$, then if the following inequalities hold in $\Omega$,

$$
\begin{gathered}
{\left[r_{10} K_{10} \frac{q}{K_{1 m}{ }^{2}}+(\alpha+\pi v) \frac{Q}{\delta_{m}}\right]^{2}<\frac{1}{3} \frac{r_{10}}{K_{1}\left(T^{*}\right)}\left(\delta+\alpha B^{*}\right)} \\
{\left[p+v_{1} \frac{Q \alpha_{1}}{\delta_{m} \alpha_{0}}\right]^{2}<\frac{1}{2} \frac{r_{10}}{K_{1}\left(T^{*}\right)}\left(\alpha_{0}+v_{1} B^{*}\right)} \\
{\left[\left(\pi v B^{*}+\theta_{1} \delta_{1}\right)+m_{3} \alpha B^{*}\right]^{2}<\frac{4}{9} m_{3}\left(\delta+\alpha B^{*}\right)\left(\delta_{1}+v B^{*}\right)} \\
\alpha_{1}^{2}<\frac{2}{9} \frac{r_{10}\left(\delta_{1}+v B^{*}\right)^{2}\left(\alpha_{0}+v_{1} B^{*}\right)}{K_{1}\left(T^{*}\right)(\alpha+v)^{2}\left(\frac{Q}{\delta_{m}}\right)^{2}}
\end{gathered}
$$

$$
\text { where } m_{3}<\frac{1}{3} \frac{r_{10}\left(\delta_{1}+v B^{*}\right)}{K_{1}\left(T^{*}\right)(\alpha+v)^{2}\left(\frac{Q}{\delta_{m}}\right)^{2}}
$$

$E^{*}$ is nonlinearly asymptotically stable with respect to all solutions initiating in the interior of the first octant. (See Appendix-B for proof)

\section{Remarks:}

1. If $Q, \alpha, \alpha_{1}, \pi$ and $\delta_{1}$ are very small, then the possibility of satisfying conditions (22) - (29) is more plausible showing that these parameters have destabilizing effect on the system.

2. If $\alpha=0, \alpha_{1}=0, \pi=0$ and $\delta_{1}=0$, then the conditions (24), (25), (28) and (29) are satis fied automatically.

The above analysis imply that as the rate of introduction of toxicants in the environment increases, then under certain conditions the densities of resource biomass and resource dependent species decrease and settle down to their respective equilibrium levels. It is pointed here that the magnitude of equilibrium of species would mainly depend upon the resource biomass density affected by intermediate toxic product formed inside the biomass due to some metabolic changes. The density of resource biomass decreases as cumulative concentration of toxicants in the environment increases and it may even tend to zero for very high concentration of toxicants and then the species dependent on it may not survive.

\section{Numerical Simulations}

In this section, we analyse the model(1) - (5) nu merically with the help of MAPLE 7.0 to study the behaviour of the systemfor different values of para meters. For this we assume that,

$$
\begin{aligned}
& r\left(U_{1}\right)=r_{10}-a_{1} U_{1}, \quad K_{1}(T)=K_{10}-b_{1} T \\
& r(B)=r_{20}+a_{2} B, \quad K_{2}(T)=K_{20}-b_{2} T
\end{aligned}
$$

Now we consider the following set of parameter values,

$$
\begin{aligned}
Q= & 5, a_{1}=0.01, r_{10}=12, b_{1}=0.3, m_{1}=1.5, \\
\pi= & 0.0015, \delta=0.9, \alpha=0.6, \alpha_{1}=0.01, v=0.65 \\
& \alpha_{0}=0.05, K_{10}=6.75, r_{20}=10, a_{2} 0.07, \\
& b_{2}=0.2, K_{20}=8, \theta_{1}=0.2, v_{1}=0.1
\end{aligned}
$$

Equilibrium values of different variable in $E^{*}$ are obtained as,

$$
\begin{aligned}
& B^{*}=6.4344695, N^{*}=8.1405519, T^{*}=1.0515293, \\
& U^{*}=0.9263452, U_{1}^{*}=0.0133585
\end{aligned}
$$

Eigen values of the matrix corresponding to $E^{*}$ are given by,

$$
\begin{aligned}
& -10.45041287,-12.30080454,-4.580458550, \\
& -4.261707507,-0.6934297045
\end{aligned}
$$

Since all the eigen values corresponding to $E^{*}$ are negative and therefore $E^{*}$ is locally asymptotically stable.

The nonlinear asymptotic stability behaviour of $E^{*}$ in $T-U$ and $N-B$ plane is shown in the figure 1 and figure 2 respectively. In these figures, it is shown that the trajectories started at any point in the region approaches the equilibrium point $E^{*}$ showing that the equilibrium is nonlinearly asymptotically stable. In figures $3,4,5$, the variation of the densities of biomass $(B)$, resource dependent species $(N)$ and the concentration of intermediate toxic product $\left(U_{1}\right)$ is shown with time at different values of rate of emission of toxicants in the environment i.e. at $Q=5,10,15$ respectively. From these figures, it is observed that the densities of biomass and species dependent on it decrease as the rate of introduction of toxicants increases and settle down to their respective equilibrium levels, while the concentration of intermediate toxic product increases. In figures 6 and 7, the variation of the densities of biomass $(B)$ and resource dependent species $(N)$ is shown with time at different values of rate of formation of intermediate toxic product $\left(U_{1}\right)$ i.e. at $\alpha_{1}=0.01,5.01,10.01$ respectively. From these figures, it can be seen that the densities of biomass and species dependent on it decrease as the rate of formation of intermediate toxic product increases and settle down to their respective equilibrium levels. In figure 8 , it is shown that if the growth of resource biomass increases, the density of species dependent on it also increases. 


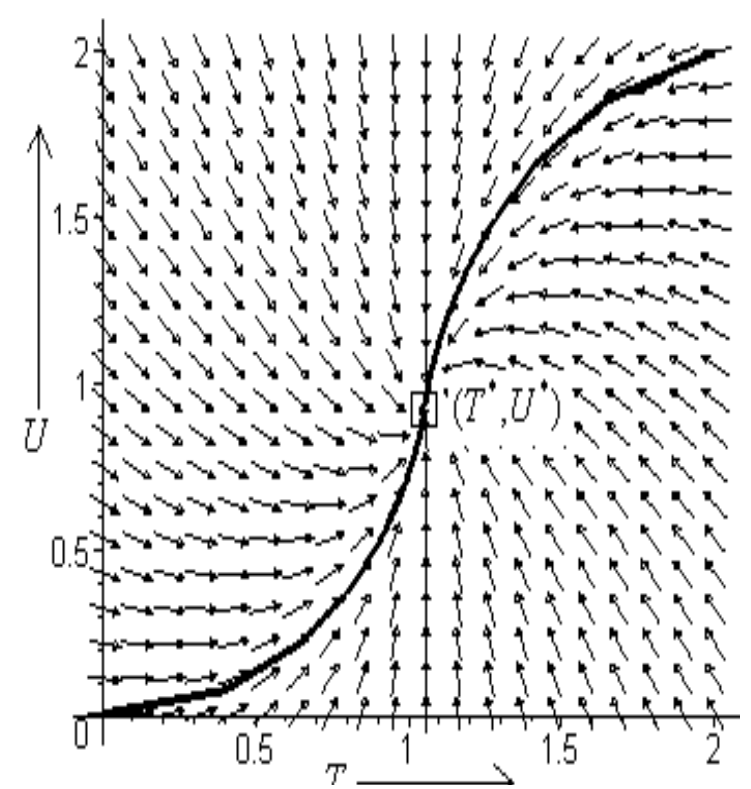

Figure 1. Nonlinear stability in $T-U$ plane

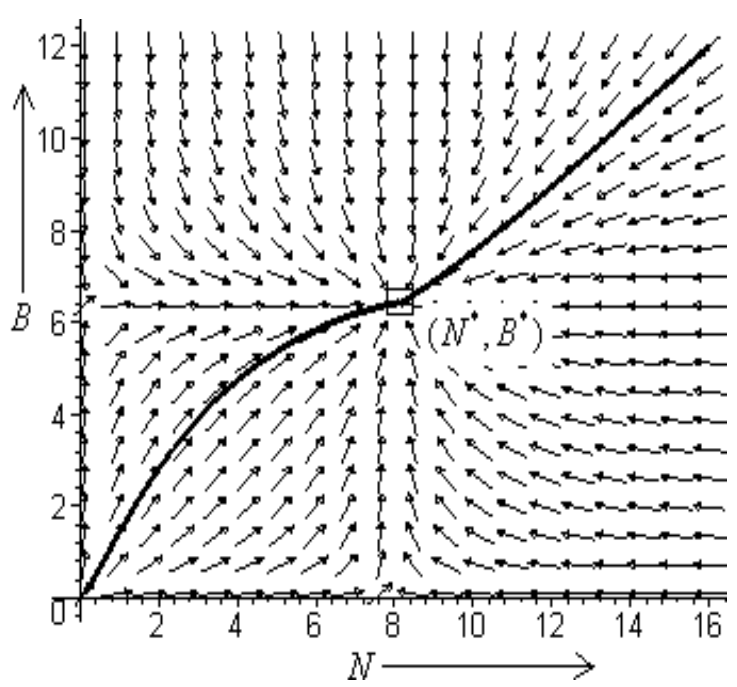

Figure 2. Nonlinear stability in $N-B$ plane

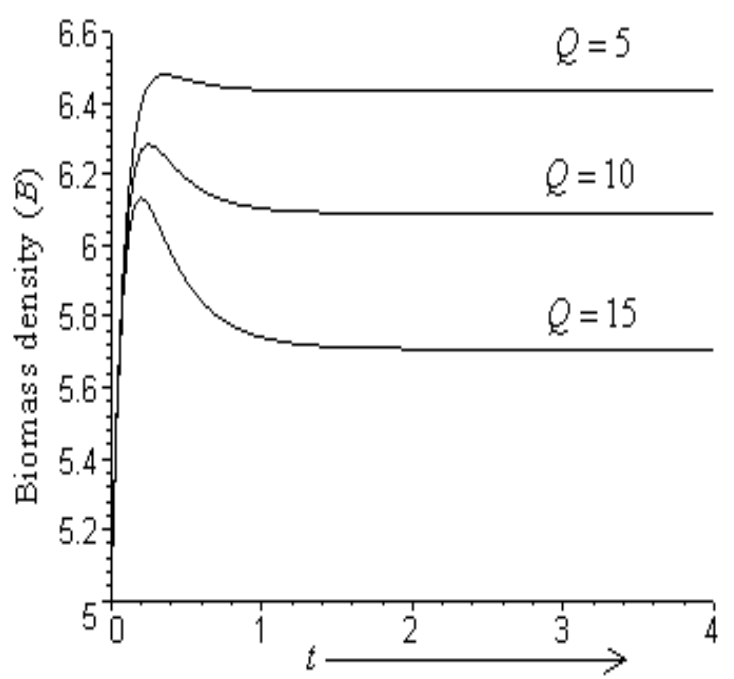

Figure 3. Variation of biomass density $B$ with time ' $t$ ' for different values of $Q$

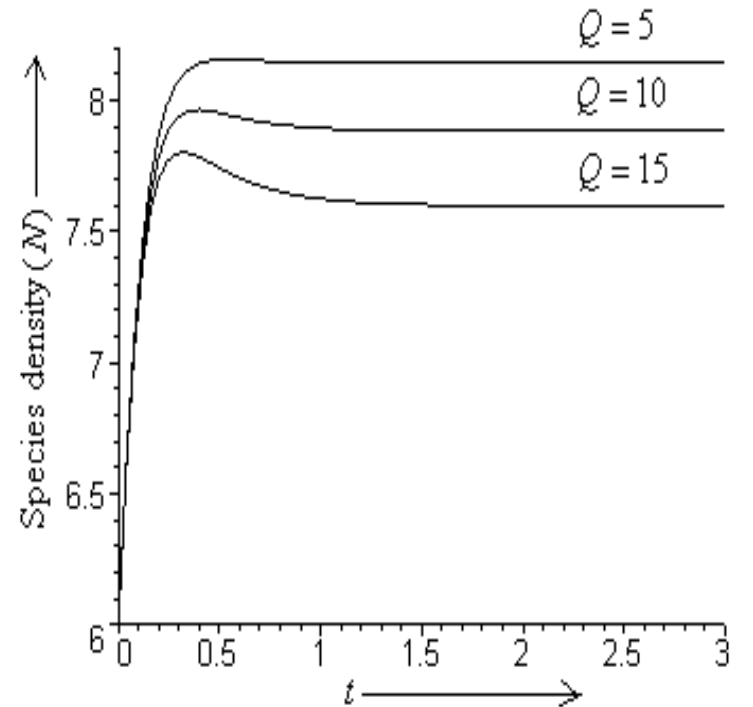

Figure 4. Variation of density of resource dependent species $N$ with time ' $t$ ' for different values of $Q$

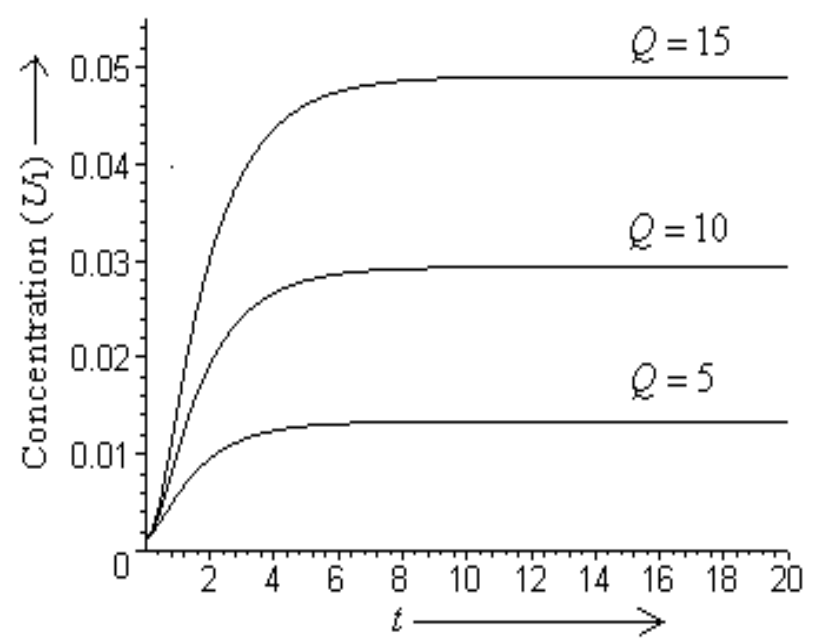

Figure 5. Variation of concentration of intermediate toxic product $U_{1}$ with time ' $t$ ' for different values of $Q$

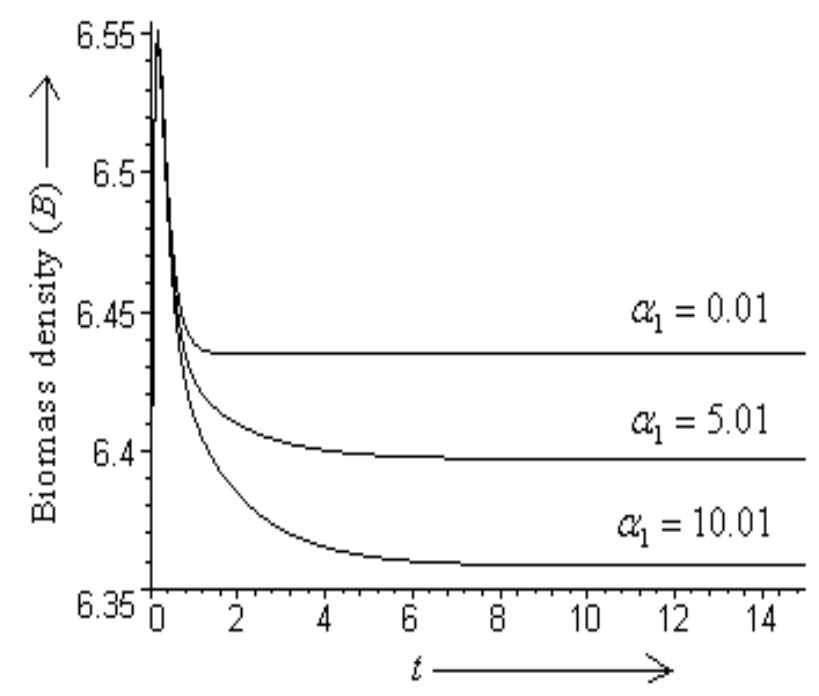

Figure 6. Variation of biomass density $B$ with time ' $t$ ' for different values of $\alpha_{1}$ 


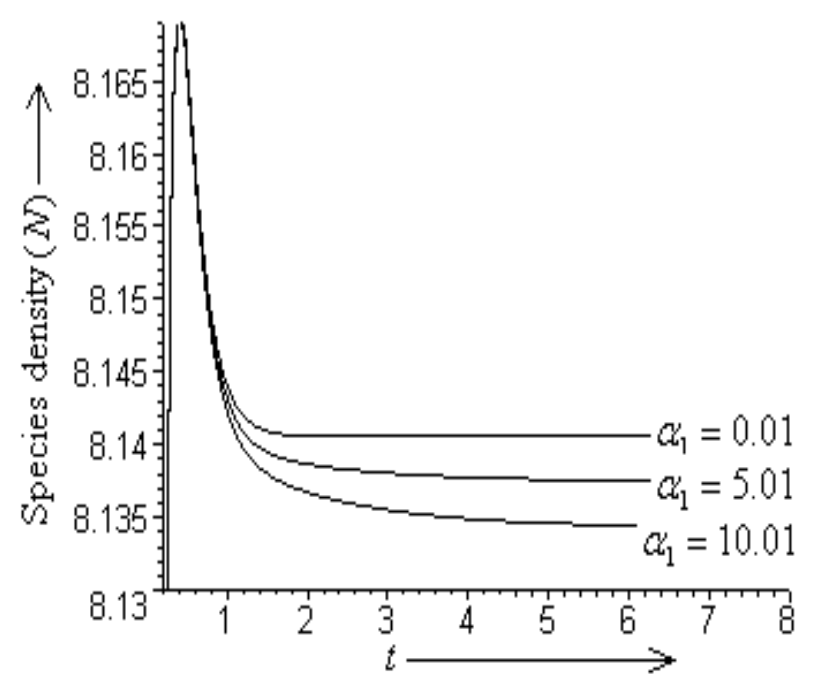

Figure 7. Variation of density of resource dependent species $N$ with time ' $t$ ' for different values of $\alpha_{1}$

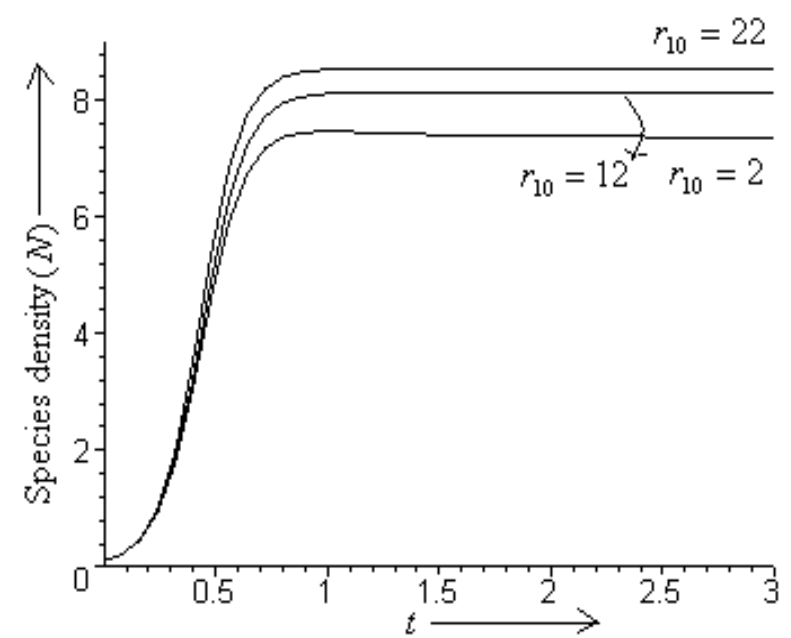

Figure 8. Variation of density of resource dependent species $N$ with time ' $t$ ' for different values of $r_{10}$

\section{Conclusions}

In this paper, we have proposed a nonlinear mathematical model to study the effect of intermed iate toxic product on the survival of a resource dependent species living in a polluted environment. It is assumed that the growth of resource biomass is affected by the intermediate to xic product formed inside the resource biomass due to some metabolic reactions when toxicants present in the environment are uptaken by resource biomass. This affected resource biomass by the intermediate toxic product then affects the resource dependent species. The model is analysed using stability theory of differential equations and computer simulations. It is shown that densities of resource biomass and species dependent on it decrease as the rate of introduction of toxicants increases in the environment and settle down to their respective equilibrium levels while the concentration of intermediate toxic product increases. Further, as the rate of formation of intermediate toxic product increases, the densities of resource biomass and the species dependent on it also decrease and their magnitudes are less than their respective densities when they are not affected by to xicant. If the rate of emission of toxicants in the environment is large enough, then under certain conditions the resource biomass may become extinct due to increased level of intermediate toxic product affecting the gro wth of bio mass and as such the species dependent on it may not survive. It is also observed that the density of species dependent on resource biomass increases if the growth of resource biomass increases. Thus, if the formation of intermediate to xic product is restricted by way of controlling the emission of toxicants in the environment, the resulting growth of resource biomass would lead to survival of species dependent on it.

\section{APPENDIX-A}

\section{Proof of the Theorem 1.}

(i) The variational matrix corresponding to $E_{0}\left(0,0, \frac{Q}{\delta}, 0,0\right)$ is given by,

$$
M_{0}=\left[\begin{array}{ccccc}
r_{01} & 0 & 0 & 0 & 0 \\
0 & r_{20} & 0 & 0 & 0 \\
-\alpha \frac{Q}{\delta} & 0 & -\delta & \theta_{1} \delta_{1} & 0 \\
\alpha \frac{Q}{\delta} & 0 & 0 & -\delta_{1} & 0 \\
0 & 0 & 0 & \alpha_{1} & -\alpha_{0}
\end{array}\right]
$$

It can be seen that the two eigen values $r_{10}, r_{20}$ of $M_{0}$ are positive, therefore $E_{0}$ is a saddle point.

The variational matrix corresponding to $E_{1}(0, \bar{N}, \bar{T}, 0,0)$ is given by,

$$
M_{1}=\left[\begin{array}{ccccc}
r_{10} & 0 & 0 & 0 & 0 \\
r_{2}^{\prime}(0) \bar{N} & r_{2}(0)-\frac{2 r_{20} \bar{N}}{K_{2}(\bar{T})} & \frac{r_{20} \bar{N}^{2}}{\left[K_{2}(T)\right]^{2}} K_{2}^{\prime}(\bar{T}) & 0 & 0 \\
-\alpha \bar{T} & 0 & -\delta & \theta_{1} \delta_{1} & 0 \\
\alpha \bar{T} & 0 & 0 & -\delta_{1} & 0 \\
0 & 0 & 0 & \alpha_{1} & -\alpha_{0}
\end{array}\right]
$$

From which we note that $E_{1}(0, \bar{N}, \bar{T}, 0,0)$ is a saddle point.

Similarly, it can also be checked that equilibrium point $E_{2}\left(\tilde{B}, 0, \tilde{T}, \tilde{U}, \tilde{U}_{1}\right)$ is unstable in $N$-direction.

(ii) Consider the following positive definite function about $E^{*}$,

$$
V=\frac{1}{2}\left[k_{0} b^{2}+k_{1} n^{2}+k_{2} \tau^{2}+k_{3} u^{2}+k_{4} u_{1}^{2}\right]
$$

where,

$$
B=B^{*}+b, N=N^{*}+n, T=T^{*}+\tau, U=U^{*}+u, U_{1}=U_{1}^{*}+u_{1}
$$

Differentiating (A1) with respect to ' $t$ ' we get

$$
\dot{V}=k_{0} b \dot{b}+k_{1} n \dot{n}+k_{2} \tau \dot{\tau}+k_{3} u \dot{u}+k_{4} u_{1} \dot{u}_{1}
$$

Now using the linearized system of the model (1) - (5) corresponding to $E^{*}\left(B^{*}, N^{*}, T^{*}, U^{*}, U_{1}^{*}\right)$ in (A2), we have, 


$$
\begin{aligned}
& \dot{V}=-k_{0} \frac{r_{10} B^{*}}{K_{1}\left(T^{*}\right)} b^{2}-k_{1} \frac{r_{20} N^{*}}{K_{2}\left(T^{*}\right)} n^{2}-k_{2}\left(\alpha B^{*}+\delta\right) \tau^{2} \\
& \quad-k_{3}\left(\delta_{1}+v B^{*}\right) u^{2}-k_{4}\left(\alpha_{0}+v_{1} B^{*}\right) u_{1}^{2} \\
& +k_{2} r_{2}^{\prime}\left(B^{*}\right) N^{*} b n \\
& +\left[k_{0} \frac{r_{10} B^{* 2}}{\left[K_{1}(T)\right]^{2}} K_{1}^{\prime}\left(T^{*}\right)-k_{2}\left(\alpha T^{*}-\pi v U^{*}\right)\right] b \tau \\
& +k_{3}\left(\alpha T^{*}-v U^{*}\right) b u+\left[k_{0} r_{1}^{\prime}\left(U_{1}^{*}\right) B^{*}-k_{4} v_{1} U_{1}^{*}\right] b u_{1} \\
& +k_{1} \frac{r_{20} N^{* 2}}{\left[K_{2}(T)\right]^{2}} K_{2}^{\prime}\left(T^{*}\right) n \tau \\
& +\left[k_{2}\left(\pi v B^{*}+\theta_{1} \delta_{1}\right)+k_{3} \alpha B^{*}\right] \tau u+k_{4} \alpha_{1} u u_{1}
\end{aligned}
$$

Now $\dot{V}$ will be negative definite, if the following inequalities are satis fied,

$$
\begin{aligned}
& k_{1}\left[r_{2}^{\prime}\left(B^{*}\right) N^{*}\right]^{2}<\frac{1}{2} k_{0} \frac{r_{10} B^{*}}{K_{1}\left(T^{*}\right)} \frac{r_{20} N^{*}}{K_{2}\left(T^{*}\right)} \\
& {\left[k_{0} \frac{r_{10} B^{* 2}}{\left[K_{1}(T)\right]^{2}} K_{1}^{\prime}\left(T^{*}\right)-k_{2}\left(\alpha T^{*}-\pi v U^{*}\right)\right]^{2}} \\
& <\frac{1}{3} k_{0} k_{2} \frac{r_{10} B^{*}}{K_{1}\left(T^{*}\right)}\left(\alpha B^{*}+\delta\right) \\
& {\left[k_{3}\left(\alpha T^{*}-v U^{*}\right)\right]^{2}<\frac{1}{3} k_{0} k_{3} \frac{r_{10} B^{*}}{K_{1}\left(T^{*}\right)}\left(\delta_{1}+v B^{*}\right)} \\
& {\left[k_{0} r_{1}^{\prime}\left(U_{1}^{*}\right) B^{*}-k_{4} v_{1} U_{1}^{*}\right]^{2}<\frac{1}{2} k_{0} k_{4} \frac{r_{10} B^{*}}{K_{1}\left(T^{*}\right)}\left(\alpha_{0}+v_{1} B^{*}\right)} \\
& {\left[k_{1} \frac{r_{20} N^{* 2}}{\left[K_{2}(T)\right]^{2}} K_{2}^{\prime}\left(T^{*}\right)\right]^{2}<\frac{2}{3} k_{1} k_{2} \frac{r_{20} N^{*}}{K_{2}\left(T^{*}\right)}\left(\alpha B^{*}+\delta\right)} \\
& {\left[k_{2}\left(\pi v B^{*}+\theta_{1} \delta_{1}\right)+k_{3} \alpha B^{*}\right]^{2}<\frac{4}{9} k_{2} k_{3}\left(\alpha B^{*}+\delta\right)\left(\delta_{1}+v B^{*}\right)} \\
& {\left[k_{4} \alpha_{1}\right]^{2}<\frac{2}{3} k_{3} k_{4}\left(\delta_{1}+v B^{*}\right)\left(\alpha_{0}+v_{1} B^{*}\right)} \\
& k_{1}<\min \left\{\begin{array}{l}
\frac{1}{2} \frac{r_{10} B^{*}}{K_{1}\left(T^{*}\right)} \frac{r_{20}}{K_{2}\left(T^{*}\right)} \frac{1}{\left[r_{2}^{\prime}\left(B^{*}\right)\right]^{2} N^{*}}, \\
\frac{2}{3}\left(\delta+\alpha B^{*}\right) \frac{K_{2}^{3}\left(T^{*}\right)}{r_{20} N^{* 3} K_{2}^{\prime 2}\left(T^{*}\right)}
\end{array}\right\} \\
& k_{3}<\frac{1}{3} \frac{r_{10} B^{*}\left(\delta_{1}+v B^{*}\right)}{K_{1}\left(T^{*}\right)\left(\alpha T^{*}+v U^{*}\right)^{2}}
\end{aligned}
$$

$\dot{V}$ will be negative definite provided the conditions (22) (25) are satisfied, showing that $V$ is a Liapunov function and hence $E^{*}$ is locally asymptotically stable.

\section{APPENDIX-B}

\section{Proof of the Theorem 2.}

Consider the following positive definite function about $E^{*}$,

$U=m_{0}\left[B-B^{*}-B^{*} \log \frac{B}{B^{*}}\right]+m_{1}\left[N-N^{*}-N^{*} \log \frac{N}{N^{*}}\right]$

$+\frac{1}{2} m_{2}\left(T-T^{*}\right)^{2}+\frac{1}{2} m_{3}\left[U-U^{*}\right]^{2}+\frac{1}{2} m_{4}\left[U_{1}-U_{1}^{*}\right]^{2}$

where the constants $m_{i}(i=0,1,2,3,4)$ can be chosen appropriately.

On differentiation we get,

$$
\begin{gathered}
\dot{U}=m_{0}\left(B-B^{*}\right) \frac{1}{B} \frac{d B}{d t}+m_{1}\left(N-N^{*}\right) \frac{1}{N} \frac{d N}{d t}+m_{2}\left[T-T^{*}\right] \frac{d T}{d t} \\
+m_{3}\left[U-U^{*}\right] \frac{d U}{d t}+m_{4}\left[U_{1}-U_{1}^{*}\right] \frac{d U_{1}}{d t}
\end{gathered} \begin{gathered}
\dot{U}=m_{0}\left(B-B^{*}\right)\left[\begin{array}{c}
{\left[U_{1}-U_{1}^{*}\right] \xi_{1}\left(U_{1}\right)-r_{10} B \eta_{1}(T)\left(T-T^{*}\right)} \\
-\frac{r_{10}}{K_{1}\left(T^{*}\right)}\left(B-B^{*}\right)
\end{array}\right] \\
+m_{1}\left(N-N^{*}\right)\left[\begin{array}{l}
\left.\left[B-B^{*}\right] \xi_{2}(B)-r_{20} N \eta_{2}(T)\left(T-T^{*}\right)\right] \\
-\frac{r_{20}}{K_{2}\left(T^{*}\right)}\left(N-N^{*}\right)
\end{array}\right] \\
+m_{2}\left(T-T^{*}\right)\left[\begin{array}{l}
-\left(\delta+\alpha B^{*}\right)\left[T-T^{*}\right]-(\alpha T-\pi v U)\left(B-B^{*}\right) \\
+\left(\theta_{1} \delta_{1}+\pi v B^{*}\right)\left(U-U^{*}\right)
\end{array}\right] \\
+m_{3}\left(U-U^{*}\right)\left[\begin{array}{l}
\alpha T-v U)\left(B-B^{*}\right)+\alpha B^{*}\left[T-T^{*}\right] \\
-\left(\delta_{1}+v B^{*}\right)\left(U-U^{*}\right)
\end{array}\right] \\
+m_{4}\left(U_{1}-U_{1}^{*}\right)\left[\begin{array}{l}
\alpha_{1}\left(U-U^{*}\right)-\left(\alpha_{0}+v_{1} B^{*}\right)\left(U_{1}-U_{1}^{*}\right) \\
-v_{1} U_{1}\left(B-B^{*}\right)
\end{array}\right]
\end{gathered}
$$

where

$$
\begin{gathered}
\xi_{1}\left(U_{1}\right)=\left\{\begin{array}{cc}
\frac{r_{1}\left(U_{1}\right)-r_{1}\left(U_{1}^{*}\right)}{U_{1}-U_{1}^{*}}, & U_{1} \neq U_{1}^{*} \\
r_{1}^{\prime}\left(U_{1}\right), & U_{1}=U_{1}^{*}
\end{array}\right. \\
\eta_{1}(T)=\left\{\begin{array}{cc}
\frac{1}{K_{1}(T)}-\frac{1}{K_{1}\left(T^{*}\right)} & T \neq T^{*} \\
-\frac{K_{1}^{\prime}(T)}{K_{1}^{2}(T)}, & T=T^{*}
\end{array}\right. \\
\xi_{2}(B)=\left\{\begin{array}{cc}
\frac{r_{2}(B)-r_{2}\left(B^{*}\right)}{B-B^{*}}, & B \neq B^{*} \\
\eta_{2}(T) & =\left\{\begin{array}{cc}
\frac{r_{2}^{\prime}(B),}{K_{2}(T)}-\frac{1}{K_{2}\left(T^{*}\right)} & B=B^{*} \\
T-T^{*} & T \neq T^{*}
\end{array}\right. \\
-\frac{K_{2}^{\prime}(T)}{K_{2}^{2}(T)}, & T=T^{*}
\end{array}\right.
\end{gathered}
$$




$$
\begin{gathered}
\dot{U}=-m_{0} \frac{r_{10}}{K_{1}\left(T^{*}\right)}\left(B-B^{*}\right)^{2}-m_{1} \frac{r_{20}}{K_{2}\left(T^{*}\right)}\left(N-N^{*}\right)^{2} \\
-m_{2}\left(\delta+\alpha B^{*}\right)\left[T-T^{*}\right]^{2} \\
-m_{3}\left(\delta_{1}+v B^{*}\right)\left(U-U^{*}\right)^{2}-m_{4}\left(\alpha_{0}+v_{1} B^{*}\right)\left(U_{1}-U_{1}^{*}\right)^{2} \\
+m_{1} \xi_{2}(B)\left[B-B^{*}\right]\left(N-N^{*}\right) \\
-\left[m_{1} r_{10} B \eta_{1}(T)+m_{2}(\alpha T-\pi v U)\right]\left(B-B^{*}\right)\left(T-T^{*}\right) \\
+m_{3}(\alpha T-v U)\left(B-B^{*}\right)\left(U-U^{*}\right) \\
+\left[m_{0} \xi_{1}\left(U_{1}\right)-m_{4} v_{1} U_{1}\right]\left(B-B^{*}\right)\left(U_{1}-U_{1}^{*}\right) \\
-m_{1} r_{20} N \eta_{2}(T)\left(N-N^{*}\right)\left(T-T^{*}\right) \\
+\left[m_{2}\left(\theta_{1} \delta_{1}+\pi v B^{*}\right)+m_{3} \alpha B^{*}\right]\left(T-T^{*}\right)\left(U-U^{*}\right) \\
+m_{4} \alpha_{1}\left(U-U^{*}\right)\left(U_{1}-U_{1}^{*}\right)
\end{gathered}
$$

Now $\dot{U}$ will be negative definite under the following sufficient conditions,

$$
\begin{gathered}
m_{1}\left[\xi_{2}\left(B^{*}\right)\right]^{2}<\frac{1}{2} m_{0} \frac{r_{10}}{K_{1}\left(T^{*}\right)} \frac{r_{20}}{K_{2}\left(T^{*}\right)} \\
{\left[m_{0} r_{10} B \eta_{1}(T)+m_{2}(\alpha T-\pi v U)\right]^{2}<\frac{1}{3} m_{0} m_{2} \frac{r_{10}}{K_{1}\left(T^{*}\right)}\left(\delta+\alpha B^{*}\right)} \\
{\left[m_{3}(\alpha T-v U)\right]^{2}<\frac{1}{3} m_{0} m_{3} \frac{r_{10}}{K_{1}\left(T^{*}\right)}\left(\delta_{1}+v B^{*}\right)} \\
{\left[m_{0} \xi_{1}\left(U_{1}\right)-m_{4} v_{1} U_{1}\right]^{2}<\frac{1}{2} m_{0} m_{4} \frac{r_{10}}{K_{1}\left(T^{*}\right)}\left(\alpha_{0}+v_{1} B^{*}\right)} \\
{\left[m_{1} r_{20} N \eta_{2}(T)\right]^{2}<\frac{2}{3} m_{1} m_{2} \frac{r_{20}}{K_{2}\left(T^{*}\right)}\left(\delta+\alpha B^{*}\right)} \\
{\left[m_{2}\left(\pi v B^{*}+\theta_{1} \delta_{1}\right)+m_{3} \alpha B^{*}\right]^{2}<\frac{4}{9} m_{2} m_{3}\left(\delta+\alpha B^{*}\right)\left(\delta_{1}+v B^{*}\right)} \\
{\left[m_{4} \alpha_{1}\right]^{2}<\frac{2}{3} m_{3} m_{4}\left(\delta_{1}+v B^{*}\right)\left(\alpha_{0}+v_{1} B^{*}\right)}
\end{gathered}
$$

Maximizing LHS and minimizing RHS and choosing $m_{0}=m_{2}=m_{4}=1$,

$$
\begin{gathered}
m_{1}<\min \left\{\frac{1}{2} \frac{r_{10}}{K_{1}\left(T^{*}\right)} \frac{r_{20}}{K_{2}\left(T^{*}\right)} \frac{1}{r^{2}}, \frac{2}{3} \frac{\left(\delta+\alpha B^{*}\right)}{K_{2}\left(T^{*}\right)\left(r_{20} N_{c} \frac{s}{K_{2 m}^{2}}\right)^{2}}\right. \\
m_{3}<\frac{1}{3} \frac{r_{10}\left(\delta_{1}+v B^{*}\right)}{K_{1}\left(T^{*}\right)(\alpha+v)^{2}\left(\frac{Q}{\delta_{m}}\right)^{2}}
\end{gathered}
$$

$\dot{U}$ will be negative definite provided the conditions (26) (29) are satisfied and hence the theorem.

\section{REFERENCES}

[1] H. I. Freedman, J.B. Shukla, Models for the effect of toxicant in single species and predator prey systems, J. Math. Biol., 30, 15-30, 1991.

[2] J. Chattopadhy ay a, Effect of toxic substance on a two species competitive sy stem, Ecol. Model., 84, 287-289, 1996.

[3] G. P. Samanta, A. Matti, Dynamical model of a single species system in a polluted environment, J. Appl. Math. Comp., 16, 231-242, 2004.

[4] T. G. Hallam, C. E. Clark, Non autonomous logistic equations as models of population in a deteriorating environment, J. Theoret. Biol., 93, 303-311, 1982.

[5] T. G. Hallam, C. E. Clark, R. R. Lassister, Effect of toxicants on populations: a qualitative approach I Equilibrium environmental exposure, Ecol. Model., 18, 291-304, 1983.

[6] W. Wang, Z. Ma, Permanence of population in a polluted environment, Math. Biosc., 122, 235-248, 1994.

[7] J.B. Shukla, B. Dubey, Simultaneous effect of two toxicants on biological species: A mathematical model, J. Biol. Syst., 4, 109-130, 1996.

[8] J. Fuhrer, P. Bungener, Effects of air pollutants on plants, Analusis, 27, 355- 360, 1999.

[9] J. B. Shukla, A. K. Agarwal, B. Dubey, P. Sinha, Existence and survival of two competing species in a polluted environment: A mathematical model, J. Biol. Syst., 9, 89-103, 2001.

[10] D. Mukherjee, Persistence and global stability of a population in a polluted environment with delay, J. Biol. Syst., 10, 225-232, 2002.

[11] B. Liu, L. Chen, Y. Zhang, The effects of impulsive toxicant input on a population in apolluted environment, J. Biol. Syst., 11, 265-274, 2003.

[12] F. Tao, B. Liu, Dynamic behaviors of a single-species population model with birthpulses in a polluted environment, Rocky Mountain J. Math., 38, 1663-1684, 2008.

[13] S. Y. Woo, Forest decline of the world: A linkage with air pollution and global warming, African J. Biotech., 8, 7409-7414, 2009.

[14] A. Yagi, T. V. Ton, Dynamics of a stochastic predator-prey population, Appl. Math. Comp., 218, 3100-3109, 2011.

[15] B. Dubey, J. Hussain, Nonlinear models for the survival of two competing species dependent on resource in industrial environments, Nonlinear Anal.: TMA, 4, 21- 44, 2003.

[16] J.B. Shukla, Shalini Sharma, B. Dubey, P. Sinha, Modeling the survival of a resource-dependent population: Effects of toxicants (pollutants) emitted from external sources as well as formed by its precursors, Nonlinear Anal.: RWA, 54-70, 2009.

[17] B. Dubey, B. Das, Models for the survival of species dependent on resource in industrial environments, J. Math. Anal. Appl., 231, 374-396, 1999.

[18] B. Dubey, J. Hussain, Modeling the survival of species dependent on a resource in a polluted environment, Nonlinear Anal.: RWA, 7, 187-210, 2006. 
[19] B. Dubey, A model for the effect of pollutant on human population dependent on a resource with environmental and health policy, J. Biol. Syst., 18, 571-592, 2010.

[20] Jiwei He, Ke Wang, The survival analysis for a population in a polluted environment, Nonlinear Anal.: RWA, 10, 1555-1571, 2009.

[21] H.P. Liu, Z. Ma, The threshold for survival for system of two species in a polluted environment, J. Math. Biol., 30, 49-61, 1991.

[22] R. Naresh, S. Sundar, J.B. Shukla, Modelling the effect of an intermediate toxic product formed by uptake of a toxicant on plant biomass, Appl. Math. Comp., 182, 151-160, 2006.

[23] H.I. Freedman, J.W.H. So, Global stability and persistence of simple food chains, Math. Biosc., 7, 69-86, 1985. 